\title{
Hypoxia Increases KIAA1199/CEMIP Expression and Enhances Cell Migration in Pancreatic Cancer
}

\section{Takuya Oba ( $\nabla$ t.oba0205@gmail.com )}

University of Occupational and Environmental Health Japan

\section{Norihiro Sato}

University of Occupational and Environmental Health Japan

\section{Yasuhiro Adachi}

University of Occupational and Environmental Health Japan

\section{Takao Amaike}

University of Occupational and Environmental Health Japan

\section{Yuzan Kudo}

University of Occupational and Environmental Health Japan

\section{Atsuhiro Koga}

Kyushu Rosai Hospital

\section{Shiro Kohi}

University of Occupational and Environmental Health Japan

\section{Keiji Hirata}

University of Occupational and Environmental Health Japan

\section{Research Article}

Keywords: Hypoxia, pancreatic ductal adenocarcinoma, hyaluronan, KIAA1199

Posted Date: February 23rd, 2021

DOI: https://doi.org/10.21203/rs.3.rs-219524/v1

License: (c) (i) This work is licensed under a Creative Commons Attribution 4.0 International License.

Read Full License 


\section{Abstract}

Pancreatic ductal adenocarcinoma (PDAC) is characterized by dense desmoplasia and hypoxic microenvironment. We previously demonstrated that hyaluronan (HA), especially low-molecular weight HA, provides a favorable microenvironment for progression of PDAC. However, the effect of hypoxia on HA metabolism is unknown. Using quantitative real-time RT-PCR and Western blot analysis, we analyzed changes in expression of HA-synthesizing enzymes (HAS2, HAS3) and HA-degrading enzymes (HYAL1, KIAA1199/CEMIP) in PDAC cell lines under hypoxic condition. Hypoxia increased mRNA and protein expression of KIAA1199, whereas it decreased expression of HYAL1. Expression of HAS2 and HAS3 remained unchanged in response to hypoxia. The effect of KIAA1199 on hypoxia-induced cell migration was determined by transwell migration assay and small-interfering RNA (siRNA). Hypoxia enhanced migratory ability of PDAC cells, which was inhibited by knockdown of KIAA1199 expression. We also used immunohistochemistry to analyze Hypoxia Inducible Factor (HIF) 1a and KIAA1199 protein expression in PDAC tissues. There was a significant immunohistochemically positive correlation between KIAA1199 and HIF1a. These findings suggest that hypoxia-induced KIAA1199 expression may contribute to enhanced motility in PDAC.

\section{Introduction}

Pancreatic ductal adenocarcinoma (PDAC) is one of the deadliest diseases worldwide, with the lowest survival rate among all cancer types ${ }^{1}$. PDAC is characterized by a dense desmoplastic stroma enriched with hyaluronan $(\mathrm{HA})^{2,3}$.

HA is produced by HA-synthesizing enzymes (HASs) and degraded into smaller fragments by hyaluronidases (HYALs) ${ }^{4-6}$. HA plays a critical role in a variety of malignant behavior including cell proliferation, migration, invasion, metastasis, angiogenesis, and resistance to drug delivery ${ }^{7-12}$. In particular, low-molecular-weight HA (LMW-HA, 100kDa or less) has been suggested to be essential for cancer progression ${ }^{13,14}$.

KIAA1199 was newly identified as one of the HA degradation enzymes. Previous studies have shown that KIAA1199 is overexpressed in many types of cancers and associated with an aggressive phenotype ${ }^{15-19}$. Notably, KIAA1199 enhances motility of colon cancer cells and is therefore called as cell migrationinducing protein (CEMIP). We also previously demonstrated that KIAA1199 expression correlates with poor prognosis in PDAC and forced expression of KIAA1199 increases migration of PDAC cells ${ }^{20}$.

Hypoxia is one of the most common stressors encountered within the tumor microenvironment ${ }^{21}$. Previous reports showed that the average oxygenation of PDAC was markedly lower compared to normal tissue (median $\mathrm{pO}_{2}$ of tumors was between 0 and $5.3 \mathrm{mmHg}$, whereas median $\mathrm{pO}_{2}$ of normal adjacent tissue was between 9.3 and $92.7 \mathrm{mmHg})^{22}$. Tumor growth leads to hypoxia. Moreover, hypoxia itself plays an important role in accelerating tumor progression, malignancy, and treatment resistance. Hypoxia signaling is mediated by the hypoxia-inducible factors (HIFs), which are stabilized under hypoxia ${ }^{23}$. 
Increased HIF-1a and/or HIF-2a levels in biopsied specimens are associated with increased risk of mortality in some cancer types including PDAC ${ }^{24}$. Although many signaling pathways, including mitogen-activated protein kinase (MAPK) signaling, are known to be altered by hypoxic conditions in PDAC cells, the effect of hypoxia on HA metabolism is unknown. In the present study, we investigated the relationship between hypoxia and HA metabolism in PDAC.

\section{Materials And Methods}

\subsection{Cell culture and reagents}

We used 3 PDAC cell lines, BxPC-3, Panc-1 (American Type Culture Collection, Manassas, VA, USA) and SUIT2 (JCRB Cell Bank, Osaka, Japan). PDAC cell lines were maintained in RPMI-1640 medium (Life Technologies, Grand Island, NY, USA) supplemented with 10\% fetal bovine serum (FBS) (Life Technologies) and $1 \%$ streptomycin and penicillin (Life Technologies), in a $5 \% \mathrm{CO}_{2}$ incubator at $37^{\circ} \mathrm{C}$. For hypoxic conditions, the cells were exposed to $1 \% \mathrm{O}_{2}$ created using a low oxygen culture kit (BIONIX low oxygen culture kit, manufactured by Sugiyamagen Co., Ltd.) for 6 to 48 hours.

\subsection{Quantitative real-time RT-PCR}

Total RNA was isolated from cell lines using RNeasy Mini Kit (QIAGEN GmbH, Hilden, Germany) according to the manufacture's protocol. First strand cDNA was synthesized from $1.0 \mu \mathrm{g}$ of total RNA using SuperScript VILO cDNA synthesis Kit and Master Mix (Thermo Fisher Scientific Inc, Waltham, MA, USA). Real-time mRNA expression analysis of HA-related genes (HAS2, HAS3, HYAL1, and KIAA1199) and a housekeeping gene (GAPDH) for control was performed using TaqMan @ Gene Expression Assays and Step One Plus real-time PCR system (Thermo Fisher Scientific Inc.) according to the manufacture's instruction. The assay numbers for these genes were as follows: Hs00193435_m1(HAS2), Hs00193436_m1(HAS3), Hs00201046_m1(HYAL1), Hs01552124_m1 (KIAA1199); and Hs02758991_g1 (GAPDH). The relative quantification was given by the Ct values, determining the reactions for target genes and an internal control gene in all samples. ${ }^{25}$

\subsection{Western blot analysis}

The cells were harvested and total protein was extracted with PRO-PREP protein extraction solution (iNtRON Biotechnology, Gyeonggi-do, South Korea), protein concentration was determined by the BCA (bicinchoninic acid) protein assay Kit (Thermo Fisher Scientific Inc., Waltham, MA, USA). Equal amount of protein were subjected to 4-15\% Mini-PROTEAN Precast Gel (Bio-Rad, Philadelphia, PA, USA) and transferred on to PVDF membranes (ATTO, Tokyo, JAPAN). Membranes were blocked for 1 hour with $1 \%$ non-fat milk in TBST buffer at room temperature, then were incubated with antibodies against KIAA1199 (Proteintech Group, Rosemont, IL, USA) and B-actin (Proteintech Group) for overnight at $4^{\circ} \mathrm{C}$ or $1 \mathrm{~h}$ at room temperature, followed by incubation with secondary antibodies (Proteintech Group) for 1 hour at room 
temperature. The proteins were visualized using an ECL Western Blotting Detection System (GE healthcare, Buckinghamshire, England). ${ }^{25}$

\subsection{Cell migration assay}

The migratory ability of cells was determined by transwell cell migration assay using cell culture inserts equipped with a filter membrane containing $8 \mu \mathrm{m}$ pores (BD Biosciences, Franklin Lajes $\mathrm{NJ}$ ). The lower chamber was filled with RPMI 1640 containing 10\% FBS. The upper chamber was filled with $1.0 \times 10^{4}$ cells (for PANC-1) or $2.0 \times 10^{4}$ cells (for BxPC-3) in the RPMI1640 containing 1\% FBS. After 48 hours incubation in each normoxic and $1 \% \mathrm{O}_{2}$ condition, the cells remaining on the upper side of the filters were removed. The cells on the bottom surface of the membrane were stained with hematoxyline and eosin and the number of cells that had migrated to the bottom surface of the membrane were counted in five randomly selected microscopic fields in each sample. ${ }^{25}$

\section{5 siRNA targeting for KIAA1199}

The siRNA for KIAA1199 (ON-TARGET plus SMART pool Human KIAA1199 L022291-00) and negative control siRNA (ON-TARGET plus Control siRNA Non-Targeting siRNA \#1 D-001810-01-05) were purchased from GE hearthcare (Buckinghamshire, England). PDAC cells (Panc1) were transfected with 100nM siRNA using DharmaFECT 1 Transfection Reagent (GE healthcare) according to manufacture' s instructions. After 48 hours treatment, the cells were immediately used for further experiments. ${ }^{25}$

\subsection{Immunohistochemistry}

Formalin-fixed and paraffin-embedded sections were stained for KIAA1199 (1:100; Proteintech Group, Inc., Chicago, IL, USA) and HIF1a (1:50; Novus Biologicals, Inc. Littleton, CO, USA). EnVision + System- HRP Labelled Polymer Anti-Rabbit (Dako North America, Inc, Carpenteria, CA, USA) was used as secondary antibody for anti-KIAA1199 antibody, and we stained them with diaminobenzidine (Liquid DAB + Substrate Chromogen System; Dako North America, Inc.). Secondary antibody for anti-HIF1a antibody was performed by streptavidin-biotin technique using SAB-PO(M) kit (NICHIREI BIOSCIENCES INC, Japan). Sections were counterstained with hematoxylin, and then dried and mounted. The proportion of positive cells was evaluated throughout the whole tissue section and graded as follows: $0(<11 \%)$; 1 (11$40 \%) ; 2$ (41-70\%); or 3 (>70\%). The staining intensity was graded as 1 (weak), 2 (moderate), or 3 (strong). The total score was obtained by multiplying the positive proportion score by the intensity score. We then divided all cases into two groups by the high expression group (with a score ranging from 0 to 2 ) or low expression group (with a score ranging from 3 to 9 ). ${ }^{20}$

\subsection{Ethical statement}

A written informed consent was obtained from all patients who approved the use of their tissues. This study was approved by institutional review board of the University of Occupational and Environmental 
Health, Kitakyushu, Japan. All methods were performed in accordance with the relevant guidelines and regulations.

\subsection{Statistical analysis}

All statistical analyses were carried out using STATA15 (Stata, College Station, TX, USA) software. Student's $t$ test and the Chi-squared test were used for group comparison. A p value of $<0.05$ was considered statistically significant.

\section{Results}

3.1 Changes in expression of HA-synthesizing and degrading enzymes in PDAC cell lines under normoxic and hypoxic conditions

We examined changes in mRNA expressions of HA synthesizing enzymes (HAS2, HAS3) and HA degrading enzymes (HYAL1, KIAA1199) upon hypoxia (Fig.1). Of these HA-related enzymes, only KIAA1199 markedly increased under hypoxia. Expression of HAS2 and HAS3 remained unchanged and expression of HYAL1 decreased under hypoxia.

The changes in protein expression of KIAA1199 under hypoxia were also analyzed using western blot analysis. KIAA1199 protein increased in a time-dependent manner under hypoxic conditions (Fig.2 and Fig.S1). $\beta$-actin levels were used as a loading control.

\subsection{Migratory ability of PDAC cells under hypoxic conditions}

We investigated the migratory ability of PDAC cells, Panc1 and BxPC3, under hypoxia. The migratory ability significantly increased up to $400 \%$ in both cell lines by hypoxia $(p<0.05)($ Fig. 3$)$.

\subsection{Effect of KIAA1199 on migration of PDAC cells in hypoxic condition}

Our previous studies have shown that KIAA1199 strongly stimulates migration at PDAC cells. We therefore hypothesized that increased expression of KIAA1199 may play a role in enhanced migration of PDAC cells upon hypoxia. To test this, we used siRNA to knockdown KIAA1199 in PDAC cells and examined their migration under hypoxic conditions. Knockdown of KIAA1199 significantly inhibited the increased migration of PDAC cells by hypoxia $(p<0.05)$ (Fig. 4).

\subsection{Immunohistochemical analysis of KIAA1199 and HIF1a in PDAC}

We then examined the relationship between KIAA1199 and HIF1a expression, as a marker of hypoxia, using immunohistochemistry in archival tissues from 25 patients with PDAC. Representative immunostaining was shown in Figure 5. KIAA1199 expression was high expression in 13 patients (52\%) and HIF1 a was high expression in 14 patients (56\%). There was a significant positive correlation between KIAA1199 and HIF1a expression in our patient group $(p<0.05)($ Table.1). 


\section{Discussion}

In the present study, we investigated the changes in HA metabolism under hypoxic conditions in PDAC cells. The major findings were as follows: (1) Hypoxia increased mRNA and protein expression of KIAA1199 in PDAC cells. (2) Hypoxia enhances migration of PDAC cells and knockdown of KIAA1199 inhibited the enhanced migration by hypoxia. (3) There was a significant immunohistochemically positive correlation between HIF1a and KIAA1199 in PDAC tissues.

In our previous studies, we have shown that HA metabolism was accelerated in PDAC cells ${ }^{10,26-28}$. However, the expression pattern of HA-synthesizing or degradation enzymes under hypoxia in PDAC has been poorly characterized. We demonstrated, for the first time, that hypoxia markedly increased expression of KIAA1199, which is known to be involved in malignant behaviors of cancer cells, in PDAC cells. These findings suggest that changes of HA metabolism under hypoxia were associated with malignant phenotype of PDAC cells.

The exact mechanisms by which KIAA1199 promotes cancer progression remain unknown. In our previous studies, knockdown of KIAA1199 expression resulted in decreased migration, whereas forced expression of KIAA1199 resulted in increased migration, invasion, and proliferation of PDAC cells ${ }^{20,25}$. In the present study, we also demonstrate that knockdown of KIAA1199 inhibited the enhanced migration under hypoxia, suggesting a possible involvement of KIAA1199 in the aggressive phenotype under hypoxia. Our present results and these finding may provide a rationale for developing novel therapeutic interventions targeting KIAA1199 for the treatment of PDAC in future studies.

We previously identified a novel phenotype, HAMP (hyaluronan activated metabolism phenotype), characterized by activation of multiple HA metabolism cascades, in PDAC ${ }^{29}$. HAMP was defined as increased expression of multiple HA metabolism genes, including KIAA1199. However, the underlying mechanisms for increased expression of these HA metabolism genes in PDAC are unclear. One such mechanism could be tumor microenvironment (or tumor-stromal interactions) in PDAC. Our present results suggest that hypoxia could contribute to the development of HAMP phenotype by increasing the expression of KIAA1199. Further studies are needed to elucidate the relationship between hypoxia and HAMP in PDAC.

In conclusion, our present study suggests that hypoxia-induced KIAA1199 expression may contribute to enhanced motility and thus microenvironment-mediated cancer progression in PDAC.

\section{Declarations}

\section{Achnowledgements}

We thank Ms. Ueda (Research Assistant, Department of Surgery1, University of Occupational and Environmental Health, Kitakyushu, JAPAN) for her technical assistance. 


\section{Funding}

This research did not receive any specific grant from funding agencies in the public, commercial, or notfor-profit sectors.

\section{Author contributions}

Takuya Oba carried out the molecular studies and drafted the manuscript. Norihiro Sato conceived of the study, and participated in its design and coordination and helped to draft the manuscript. Atsuhiro Koga, Yasuhiro Adachi, Yuzan Kudo, Takao Amaike and Shiro Kohi participated in the molecular studies. Keiji Hirata participated in the design of the study. All authors read and approved the final manuscript.

\section{Competing interest}

The authors declare no competing interest.

\section{Data availability}

The datasets generated during and/or analysed during the current study are available from the corresponding author on reasonable request.

\section{References}

1 Vincent, A., Herman, J., Schulick, R., Hruban, R. H. \& Goggins, M. Pancreatic cancer. Lancet378, 607-620, doi:10.1016/s0140-6736(10)62307-0 (2011).

2 Mahlbacher, V., Sewing, A., Elsässer, H. P. \& Kern, H. F. Hyaluronan is a secretory product of human pancreatic adenocarcinoma cells. Eur J Cell Bio/58, 28-34 (1992).

3 Whatcott, C. J. et al. Desmoplasia in Primary Tumors and Metastatic Lesions of Pancreatic Cancer. Clin Cancer Res21, 3561-3568, doi:10.1158/1078-0432.Ccr-14-1051 (2015).

4 Itano, N. \& Kimata, K. Mammalian hyaluronan synthases. IUBMB Life54, 195-199, doi:10.1080/15216540214929 (2002).

5 Stern, R. Hyaluronan catabolism: a new metabolic pathway. Eur J Cell Bio/83, 317-325, doi:10.1078/0171-9335-00392 (2004).

6 Toole, B. P. Hyaluronan: from extracellular glue to pericellular cue. Nat Rev Cancer4, 528-539, doi:10.1038/nrc1391 (2004).

7 Itano, N., Zhuo, L. \& Kimata, K. Impact of the hyaluronan-rich tumor microenvironment on cancer initiation and progression. Cancer Sci99, 1720-1725, doi:10.1111/j.1349-7006.2008.00885.x (2008). 
8 Jacobetz, M. A. et al. Hyaluronan impairs vascular function and drug delivery in a mouse model of pancreatic cancer. Gut62, 112-120, doi:10.1136/gutjnl-2012-302529 (2013).

9 Provenzano, P. P. et al. Enzymatic targeting of the stroma ablates physical barriers to treatment of pancreatic ductal adenocarcinoma. Cancer Cel/21, 418-429, doi:10.1016/j.ccr.2012.01.007 (2012).

10 Sato, N., Kohi, S., Hirata, K. \& Goggins, M. Role of hyaluronan in pancreatic cancer biology and therapy: Once again in the spotlight. Cancer Sci107, 569-575, doi:10.1111/cas.12913 (2016).

11 Sironen, R. K. et al. Hyaluronan in human malignancies. Exp Cell Res317, 383-391, doi:10.1016/j.yexcr.2010.11.017 (2011).

12 Toole, B. P., Wight, T. N. \& Tammi, M. I. Hyaluronan-cell interactions in cancer and vascular disease. $J$ Biol Chem277, 4593-4596, doi:10.1074/jbc.R100039200 (2002).

13 Sugahara, K. N. et al. Tumor cells enhance their own CD44 cleavage and motility by generating hyaluronan fragments. J Biol Chem281, 5861-5868, doi:10.1074/jbc.M506740200 (2006).

$14 \mathrm{Wu}, \mathrm{M}$. et al. A novel role of low molecular weight hyaluronan in breast cancer metastasis. Faseb j29, 1290-1298, doi:10.1096/fj.14-259978 (2015).

15 Birkenkamp-Demtroder, K. et al. Repression of KIAA1199 attenuates Wnt-signalling and decreases the proliferation of colon cancer cells. Br J Cancer105, 552-561, doi:10.1038/bjc.2011.268 (2011).

16 Evensen, N. A. et al. Hypoxia promotes colon cancer dissemination through up-regulation of cell migration-inducing protein (CEMIP). Oncotarget6, 20723-20739, doi:10.18632/oncotarget.3978 (2015).

17 Fink, S. P. et al. Induction of KIAA1199/CEMIP is associated with colon cancer phenotype and poor patient survival. Oncotarget6, 30500-30515, doi:10.18632/oncotarget.5921 (2015).

18 Kuscu, C. et al. Transcriptional and epigenetic regulation of KIAA1199 gene expression in human breast cancer. PLoS One7, e44661, doi:10.1371/journal.pone.0044661 (2012).

19 Matsuzaki, S. et al. Clinicopathologic significance of KIAA1199 overexpression in human gastric cancer. Ann Surg Onco/16, 2042-2051, doi:10.1245/s10434-009-0469-6 (2009).

20 Koga, A. et al. KIAA1199/CEMIP/HYBID overexpression predicts poor prognosis in pancreatic ductal adenocarcinoma. Pancreatology17, 115-122, doi:10.1016/j.pan.2016.12.007 (2017).

21 Brahimi-Horn, M. C., Chiche, J. \& Pouysségur, J. Hypoxia and cancer. J Mol Med (Berl)85, 1301-1307, doi:10.1007/s00109-007-0281-3 (2007).

22 Koong, A. C. et al. Pancreatic tumors show high levels of hypoxia. Int J Radiat Oncol Biol Phys48, 919922, doi:10.1016/s0360-3016(00)00803-8 (2000). 
23 Yuen, A. \& Díaz, B. The impact of hypoxia in pancreatic cancer invasion and metastasis. Hypoxia (Auckl)2, 91-106, doi:10.2147/hp.S52636 (2014).

24 Semenza, G. L. Defining the role of hypoxia-inducible factor 1 in cancer biology and therapeutics. Oncogene29, 625-634, doi:10.1038/onc.2009.441 (2010).

25 Kohi, S., Sato, N., Koga, A., Matayoshi, N. \& Hirata, K. KIAA1199 is induced by inflammation and enhances malignant phenotype in pancreatic cancer. Oncotarget8, 17156-17163, doi:10.18632/oncotarget.15052 (2017).

26 Cheng, X. B., Kohi, S., Koga, A., Hirata, K. \& Sato, N. Hyaluronan stimulates pancreatic cancer cell motility. Oncotarget7, 4829-4840, doi:10.18632/oncotarget.6617 (2016).

$27 \mathrm{Kohi}$, S. et al. A novel epigenetic mechanism regulating hyaluronan production in pancreatic cancer cells. Clin Exp Metastasis33, 225-230, doi:10.1007/s10585-015-9771-9 (2016).

28 Sato, N., Cheng, X. B., Kohi, S., Koga, A. \& Hirata, K. Targeting hyaluronan for the treatment of pancreatic ductal adenocarcinoma. Acta Pharm Sin B6, 101-105, doi:10.1016/j.apsb.2016.01.002 (2016).

29 Kudo, Y., Kohi, S., Hirata, K., Goggins, M. \& Sato, N. Hyaluronan activated-metabolism phenotype (HAMP) in pancreatic ductal adenocarcinoma. Oncotarget10, 5592-5604, doi:10.18632/oncotarget.27172 (2019).

\section{Tables}

Table.1 Immunostaining of PDAC tissue with anti-HIF1a antibody and anti-KIAA1199 antibody

\begin{tabular}{|cccc|}
\hline & & \multicolumn{2}{c|}{ KIAA1199 } \\
& & high & low \\
\hline HIF1a & high & 10 & 4 \\
\cline { 2 - 4 } & low & 3 & 8 \\
\hline
\end{tabular}

\section{Figures}



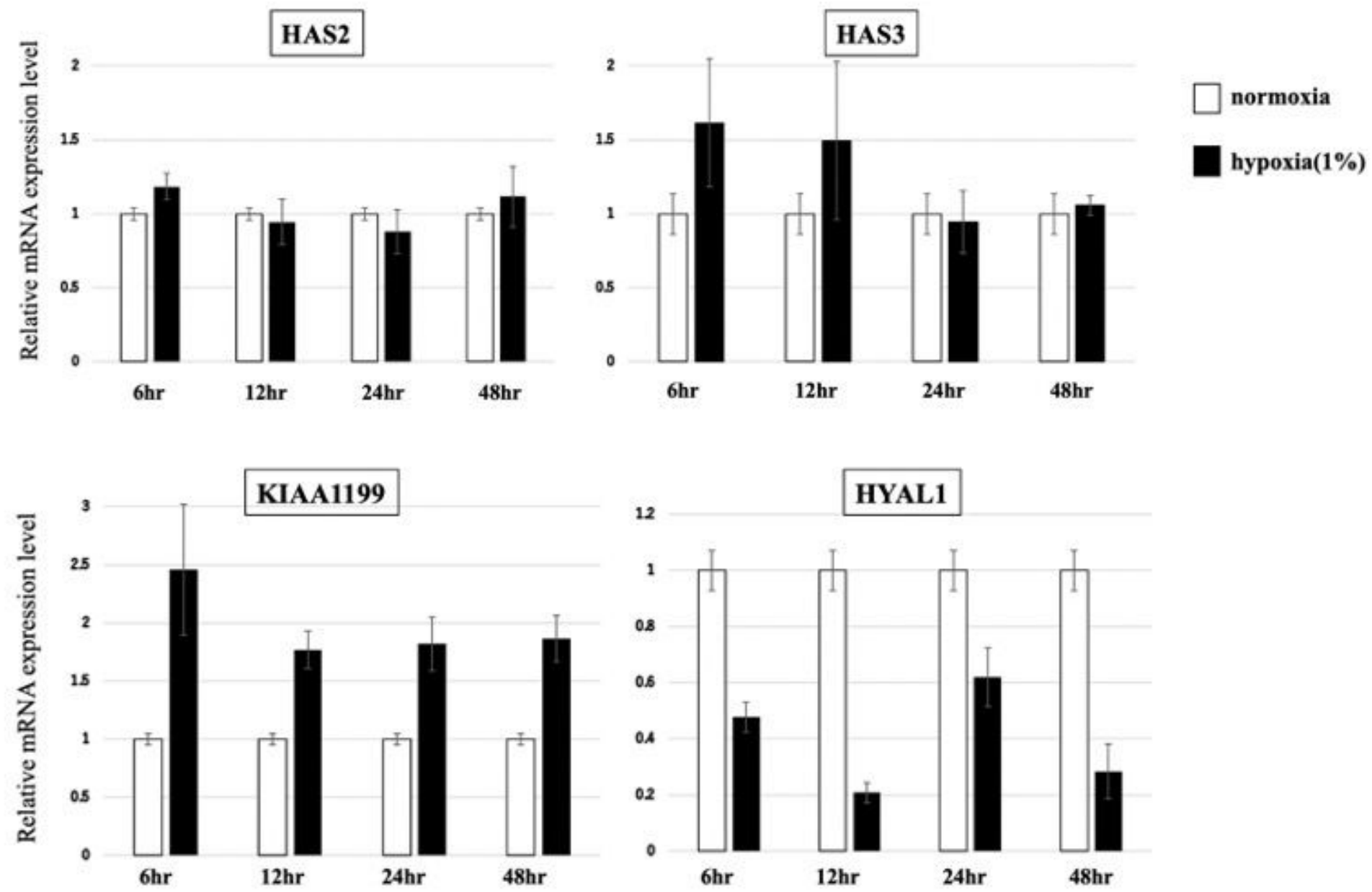

Figure 1

The changes of mRNA expression of HA synthesizing enzymes and HA degrading enzymes were shown. Expression of KIAA1199 increased under hypoxia. Expression of HAS2 and HAS3 remained unchanged and expression of HYAL1 decreased under hypoxia.

KIAA1199

$\beta$-actin

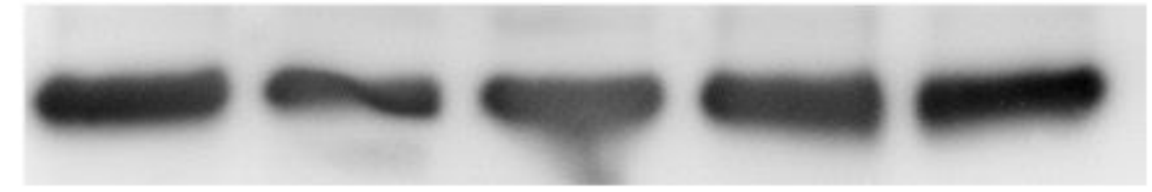

Hypoxia

$0 \mathrm{~h}$

$6 \mathrm{~h}$

$12 \mathrm{~h}$

$24 \mathrm{~h}$

$48 \mathrm{~h}$

Figure 2 
KIAA1199 protein increased in a time-dependent manner under hypoxic conditions. $\beta$-actin levels were used as a loading control.
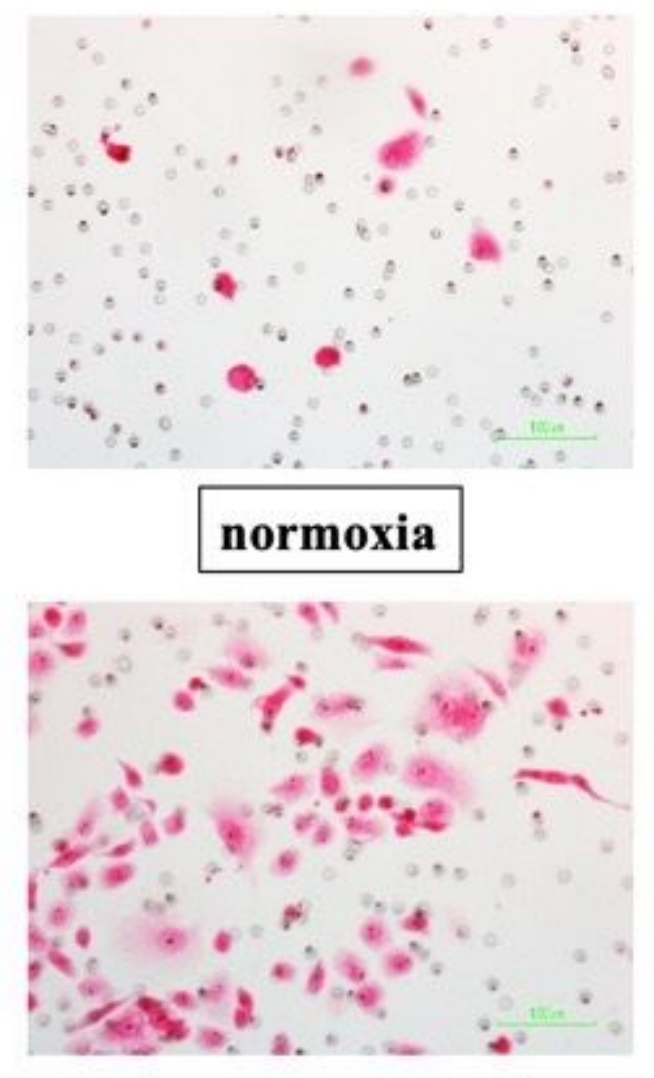

hypoxia (1\%)

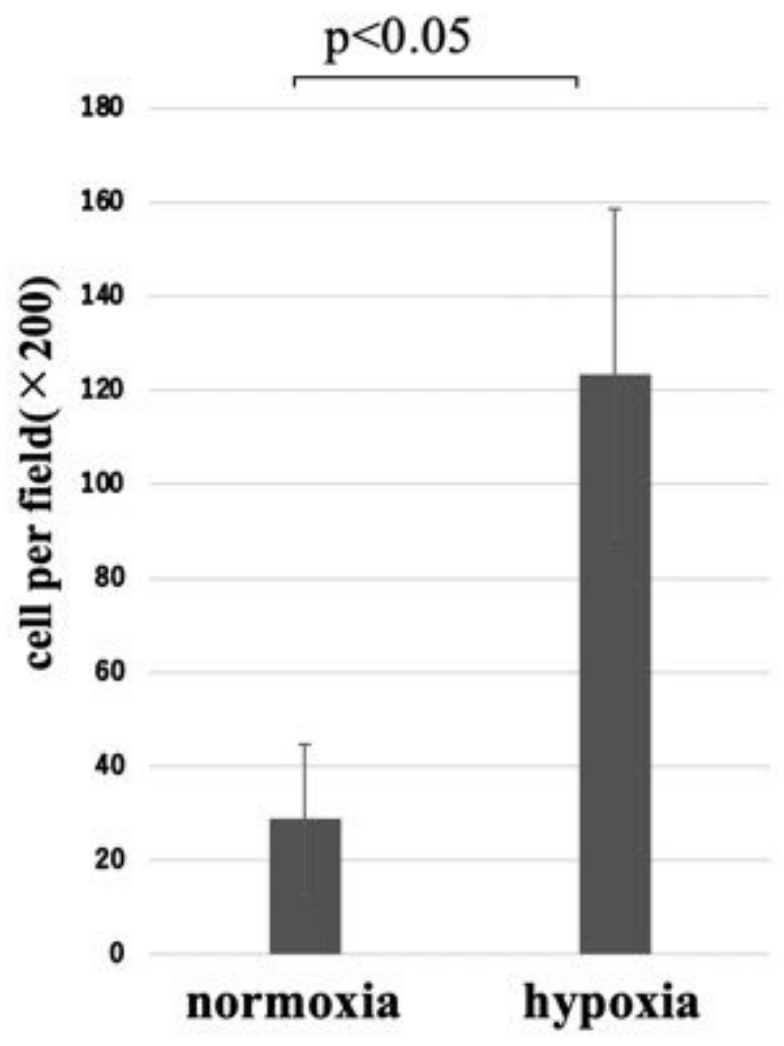

Figure 3

Cell migratory ability of PDAC cells, Panc-1, significantly increased by hypoxia. 

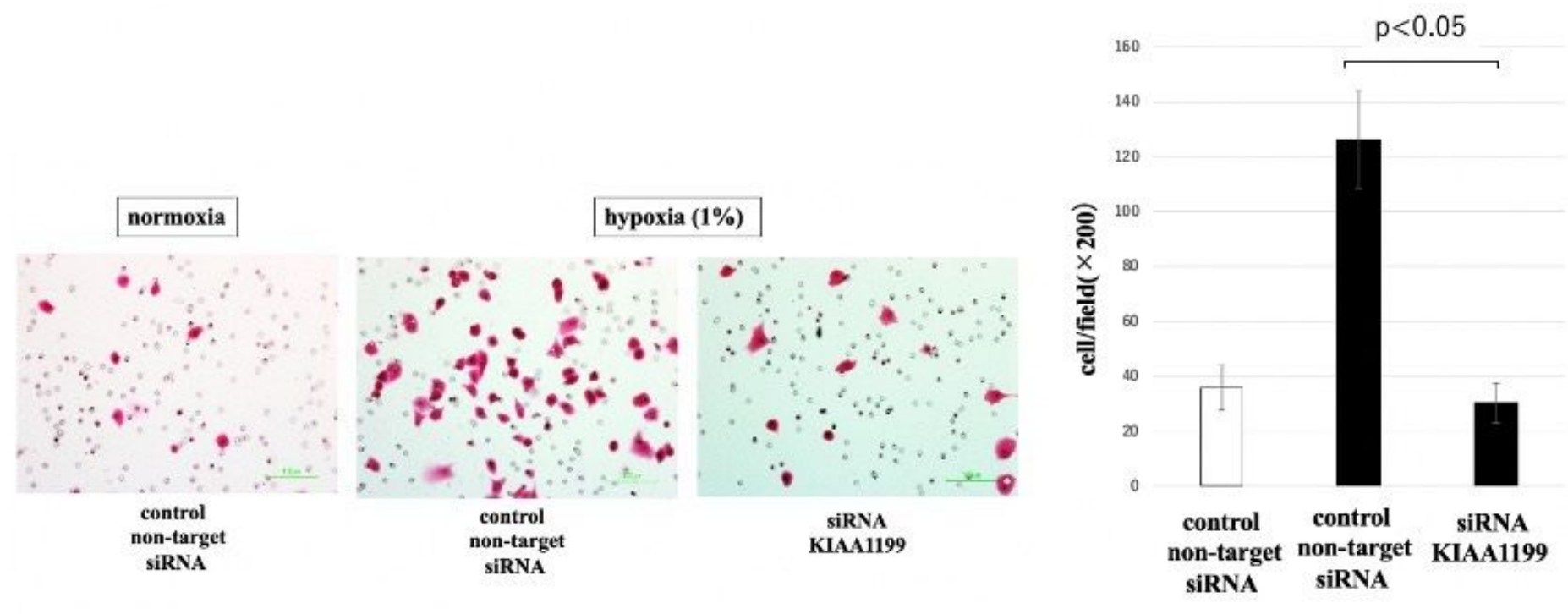

\section{Figure 4}

Cell migratory ability of PDAC cells, Panc-1, under hypoxia with/without knockdown of KIAA1199. Knockdown of KIAA1199 inhibited increased migration by hypoxia 

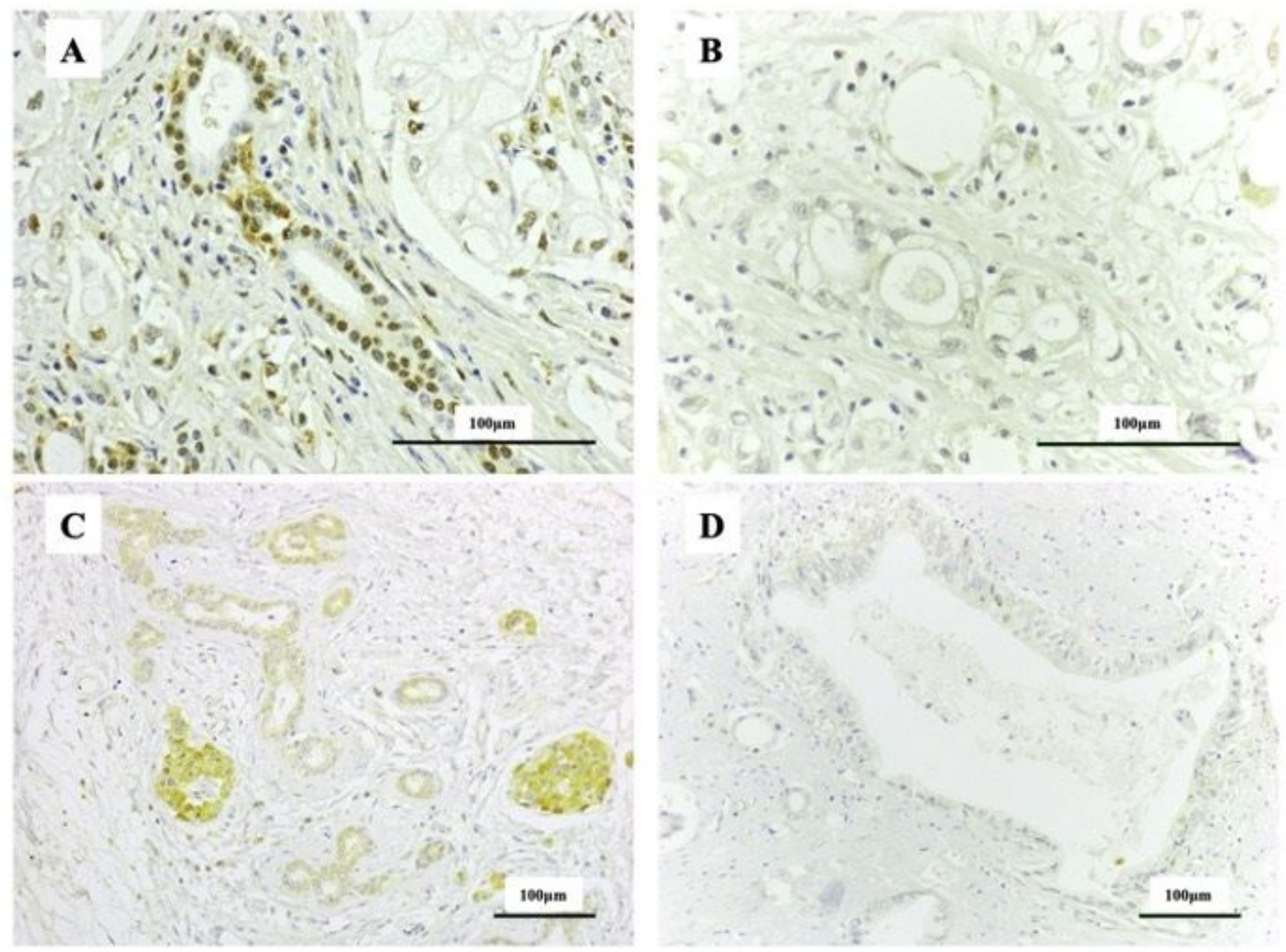

D

\section{Figure 5}

Immunostaining of PDAC tissue with anti-HIF1a antibody and anti-KIAA1199 antibody. A: A representative image of strong nucleatic labeling of PDAC cells (classified as high HIF1a expression group). B: A representative image classified as low HIF1a expression group. C: A representative image of strong cytoplastic labeling of PDAC cells (classified as high KIAA1199 expression group). D: A representative image classified as low KIAA1199 expression group. Table.1: Immunostaining of PDAC tissue with anti-HIF1a antibody and anti-KIAA1199 antibody. There was a significant positive correlation between KIAA1199 and HIF1a expression in our patient group $(p<0.05)$.

\section{Supplementary Files}

This is a list of supplementary files associated with this preprint. Click to download.

- SupplementaryFile.pdf 